\title{
Wave-like variables of a classical particle and their connections to quantum mechanics
}

DOI:

10.1088/0143-0807/38/1/015401

\section{Document Version}

Final published version

Link to publication record in Manchester Research Explorer

\section{Citation for published version (APA):}

Yang, C. (2017). Wave-like variables of a classical particle and their connections to quantum mechanics. European Journal of Physics, 38(1), [015401]. https://doi.org/10.1088/0143-0807/38/1/015401

\section{Published in:}

European Journal of Physics

\section{Citing this paper}

Please note that where the full-text provided on Manchester Research Explorer is the Author Accepted Manuscript or Proof version this may differ from the final Published version. If citing, it is advised that you check and use the publisher's definitive version.

\section{General rights}

Copyright and moral rights for the publications made accessible in the Research Explorer are retained by the authors and/or other copyright owners and it is a condition of accessing publications that users recognise and abide by the legal requirements associated with these rights.

\section{Takedown policy}

If you believe that this document breaches copyright please refer to the University of Manchester's Takedown Procedures [http://man.ac.uk/04Y6Bo] or contact uml.scholarlycommunications@manchester.ac.uk providing relevant details, so we can investigate your claim.

\section{OPEN ACCESS}


Wave-like variables of a classical particle and their connections to quantum mechanics

This content has been downloaded from IOPscience. Please scroll down to see the full text.

2017 Eur. J. Phys. 38015401

(http://iopscience.iop.org/0143-0807/38/1/015401)

View the table of contents for this issue, or go to the journal homepage for more

Download details:

IP Address: 130.88.3.181

This content was downloaded on 15/02/2017 at 12:00

Please note that terms and conditions apply.

You may also be interested in:

Quantum Mechanics: The failure of classical physics and the advent of quantum mechanics M Saleem

Quantum Information in Gravitational Fields: Quantum fields in curved spacetimes

M Lanzagorta

Magnetic Excitations and Geometric Confinement: Magnetism theory: spin models

G M Wysin

Symmetry and Collective Fluctuations in Evolutionary Games: Symmetry and collective fluctuations: large deviations and scaling in population processes

E Smith and S Krishnamurthy

Magnetic Excitations and Geometric Confinement: Magnetic vortex core motion and internal dynamics

G M Wysin

Progress in classical and quantum variational principles

C G Gray, G Karl and V A Novikov

From classical to quantum mechanics through optics

Jaume Masoliver and Ana Ros

Path integral methods for the dynamics of stochastic and disordered systems

John A Hertz, Yasser Roudi and Peter Sollich

Quasiclassical analysis of Bloch oscillations in non-Hermitian tight-binding lattices

E M Graefe, H J Korsch and A Rush 


\title{
Wave-like variables of a classical particle and their connections to quantum mechanics
}

\author{
Chen Yang \\ School of Mechanical, Aerospace and Civil Engineering, University of Manchester, \\ Manchester M13 9PL, UK \\ E-mail: chen.yang@manchester.ac.uk
}

Received 19 July 2016, revised 21 September 2016

Accepted for publication 6 October 2016

Published 25 October 2016

\begin{abstract}
In many texts, the transition from classical mechanics to quantum mechanics is achieved by substituting the action for the phase angle. The paper presents a different approach to show some connections between classical and quantum mechanics for a single particle for an audience at graduate and postgraduate levels. Firstly, it is shown that a wave equation of action can be derived under the free particle condition and the Legendre transform. The wave-like solutions of the action, Hamiltonian and momentum of the free particle are presented. Using the discrete approximation, the equation of motion of a single particle, in scalar potential field, is obtained in a similar form to Schrödinger's equation. The rest of the paper discusses the propagation, superposition of the wave-like dynamic variables and their connections to quantum mechanics. The superposition of the variables of a particle is generally distinct from the superposition of classical waves (e.g. acoustics). The quantum superposition provides a self-consistent interpretation of the wave-like solutions of the variables. Connections between the classical and quantum relations for corresponding variables are observed from the one-to-one comparisons.
\end{abstract}

Keywords: wave-like variable, particle, classical mechanics, connections, quantum mechanics

\section{Introduction}

The physical pictures of a particle and a wave are distinct and well-established in various fields of classical mechanics [1-5]. The emergence of quantum mechanics has revealed the inherent connections between the two common pictures as necessary but distinctive aspects of elementary particles [6-13]. The connections between classical particle dynamics and 
quantum mechanics have been studied and reported in the literature [1, 4, 13-30]. The transition from the Hamiltonian formalism to the Schrödinger picture has been investigated by several authors. The substitution of the action for the phase in the Hamilton-Jacobi equation has been presented in the literature [10-19]. At the same time, the influence of the free particle condition on the action itself has not received much attention. In this paper, an approach (that requires the knowledge of classical mechanics at graduate and postgraduate levels) is presented to show the connections between classical and quantum relations. It is shown that the free particle condition and the Legendre transform leads to a linear homogenous wave equation of action. The wave equation of the action provides the wave-like solution for certain dynamic variables (e.g. action, Hamiltonian and momentum) of the particle. This enables these specific dynamic variables to be expressed in a similar form to the corresponding quantum relations.

To start with, it is assumed that the status of the particle can be characterised via its dynamic variables over the space and time. From classical mechanics, a free particle requires that the velocity, Hamiltonian and momentum of the particle are invariant under time evolution. The free particle condition gives a set of first-order homogenous equations of the above dynamic variables. At the same time, the Legendre transform provides first-order relations between the action, the Hamiltonian and the momentum of a particle. Considering the two sets of first-order relations simultaneously, it can be shown that the action of a free particle is governed by a linear homogenous wave equation. This provides wave-like solutions of action, Hamiltonian and momentum for a free particle. The solutions of the free particle can be further extended to the cases with conservative potentials using an approximation approach. Equation of the motion of a single particle in scalar potential field is obtained by approximation.

The rest of the paper discusses the propagation and superposition of the wave-like dynamic variables and its connections to quantum mechanics. The propagation of wave-like dynamic variables from multiple-trajectory is investigated using Maupertuis' principle. On one hand, the superposition of the physical variable along different trajectories occurs from the feature of the wave-like solution. On the other hand, the superposition of the wave-like dynamic variables does not follow the superposition of classical waves. It is then realised that the rule of quantum superposition is a promising way to provide a self-consistent picture. This motivates further comparison of the obtained classical relations and the quantum relations of the variables. The observed one-to-one correspondences between the two sets of relations are discussed. The main points of the work are summarised in the conclusion section of the paper.

\section{Detailed procedures}

\subsection{Equation of action for a free particle}

Consider the dynamic variables of a particle that are functions of displacement $\boldsymbol{r}(t)$, velocity $\dot{\boldsymbol{r}}(t)$ and time $t$ defined in the Cartesian coordinate. For a free particle, the dynamic variables of the particle are invariant with respect to the evolution of time $\mathrm{d} t$ as in the following relations,

$$
\frac{\mathrm{d}}{\mathrm{d} t} H(\boldsymbol{r}, \boldsymbol{P}, t)=\frac{\partial H}{\partial \boldsymbol{r}} \frac{\mathrm{d} \boldsymbol{r}}{\mathrm{d} t}+\frac{\partial H}{\partial \boldsymbol{P}} \frac{\mathrm{d} \boldsymbol{P}}{\mathrm{d} t}+\frac{\partial H}{\partial t}=0
$$


and

$$
\frac{\mathrm{d}}{\mathrm{d} t} \boldsymbol{P}(\boldsymbol{r}, \dot{\boldsymbol{r}}, t)=\frac{\partial \boldsymbol{P}}{\partial \boldsymbol{r}} \frac{\mathrm{d} \boldsymbol{r}}{\mathrm{d} t}+\frac{\partial \boldsymbol{P}}{\partial \dot{\boldsymbol{r}}} \frac{\mathrm{d} \dot{\boldsymbol{r}}}{\mathrm{d} t}+\frac{\partial \boldsymbol{P}}{\partial t}=0
$$

and

$$
\frac{\mathrm{d}}{\mathrm{d} t} \dot{\boldsymbol{r}}=0,
$$

where $H$ and $\boldsymbol{P}$ denote the Hamiltonian (energy) and momentum of the particle. From the Legendre transform, the action and its relations to the Hamiltonian and momentum of a particle are,

$$
S \equiv \int L(\boldsymbol{r}, \dot{\boldsymbol{r}}, t) \mathrm{d} t=\int \boldsymbol{P} \mathrm{d} \boldsymbol{r}-\int H \mathrm{~d} t,
$$

where

$$
H=-\frac{\partial S}{\partial t}, \quad \boldsymbol{P}=\frac{\partial S}{\partial \boldsymbol{r}} .
$$

It should be noted that the first-order partial derivative of the Hamiltonian $H$ and momentum $\boldsymbol{P}$ given in equations (1) and (2) can be expressed using the partial derivatives of the action $S$. Additionally, the total derivative of the momentum in equation (1) vanishes and the acceleration term in the equation (2) vanishes under the free particle condition. Hence, a second-order differential equation of action can be constructed by substituting equation (5) into equations (1) and (2),

$$
\frac{\partial^{2} S}{\partial \boldsymbol{r} \partial t} \frac{\mathrm{d} \boldsymbol{r}}{\mathrm{d} t}+\frac{\partial^{2} S}{\partial t^{2}}=0
$$

and

$$
\frac{\partial^{2} S}{\partial \boldsymbol{r}^{2}} \frac{\mathrm{d} \boldsymbol{r}}{\mathrm{d} t}+\frac{\partial^{2} S}{\partial t \partial \boldsymbol{r}}=0
$$

The second-order mixed derivatives of action are commutative under the smoothness condition,

$$
\frac{\partial^{2} S}{\partial \boldsymbol{r} \partial t}=\frac{\partial^{2} S}{\partial t \partial \boldsymbol{r}} .
$$

Dividing equation (6) by the velocity term, substituting the rest of the equation into equation (7) and using the commutative relation in equation (8) gives a linear second-order partial differential equation with respect to the action with only pure spatial and time derivatives, that is,

$$
\frac{\partial^{2} S}{\partial \boldsymbol{r}^{2}}\left(\frac{\mathrm{d} \boldsymbol{r}}{\mathrm{d} t}\right)^{2}-\frac{\partial^{2} S}{\partial t^{2}}=0 .
$$

The Hamiltonian and momentum of the free particle at initial condition $\boldsymbol{r}=0, t=0$ are denoted by $H_{0}$ and $\boldsymbol{P}_{0}$, respectively. Using the Taylor expansions, the velocity of the free particle from the Hamiltonian and the momentum is,

$$
\frac{\mathrm{d} \boldsymbol{r}}{\mathrm{d} t}=\frac{\partial H}{\partial \boldsymbol{P}}=\frac{\partial H_{0}}{\partial \boldsymbol{P}_{0}}+\frac{\partial^{2} H_{0}}{\partial \boldsymbol{P}_{0}^{2}} \mathrm{~d} \boldsymbol{P}+\cdots .
$$

Under the free particle condition, the first term remains and the rest of the partial derivative terms vanish due to the vanishing of $\mathrm{d} \boldsymbol{P}$ with respect to time as in equation (2). Substituting 
the above expansion into equation (9) yields the linear homogenous wave equation of the action,

$$
\frac{\partial^{2} S}{\partial \boldsymbol{r}^{2}}\left(\frac{\partial H_{0}}{\partial \boldsymbol{P}_{0}}\right)^{2}-\frac{\partial^{2} S}{\partial t^{2}}=0
$$

\subsection{Plane wave solutions of dynamic variables}

From the theory of differential equation [31], the non-trivial solutions of the wave equation are in the form of plane waves and their linear superposition. Assuming the real part of the action integral is zero at initial condition $S_{\text {real }}(\boldsymbol{r}=0, t=0)=0$, the plane wave solution of the action is,

$$
S=-\mathrm{i} \bar{S} \phi(\boldsymbol{r}, t)
$$

where

$$
\phi(\boldsymbol{r}, t)=\mathrm{e}^{\mathrm{i} \theta}=\mathrm{e}^{\mathrm{i}(\boldsymbol{k} \boldsymbol{r}-\omega t)}
$$

is the phase factor, $\bar{S}$ denotes the magnitude of the action (independent of space and time) and the imaginary unit i denotes the phase shift from the initial condition. It is worth to note that for any non-zero magnitude $(\bar{S} \neq 0)$, the action follows the wave-like solutions over space and time. According to equation (5), the Hamiltonian and momentum of the free particle become,

$$
H=\mathrm{i} \bar{S} \frac{\partial \phi}{\partial t}=\bar{S} \omega \phi, \quad \boldsymbol{P}=-\mathrm{i} \bar{S} \frac{\partial \phi}{\partial \boldsymbol{r}}=\bar{S} \boldsymbol{k} \phi
$$

These describe a wave-like solution of the dynamic variables that remain unchanged during the time evolution. It can be seen that the above solutions satisfy the free particle condition as the total time derivative of the phase factor vanish identically $\mathrm{d} \phi / \mathrm{d} t=0$. Substituting the initial condition for the Hamiltonian and momentum of the free particle in equation (14), one obtains,

$$
H_{0}=\bar{S} \omega_{0}, \quad \boldsymbol{P}_{0}=\bar{S} \boldsymbol{k}_{0} .
$$

The above relations show that the magnitude of action $\bar{S}$ is independent of the Hamiltonian and momentum of the particle. They are also similar to the de Broglie relations in quantum mechanics if the magnitude of action $\bar{S}$ is replaced by the Planck's constant $\hbar$. With the aid of the above relations and from the definition of linear differential operator, the equation (14) can be further written in the linear differential operator forms as,

$$
\hat{H} \phi \equiv \mathrm{i} \bar{S} \frac{\partial \phi}{\partial t}=H_{0} \phi, \quad \hat{\boldsymbol{P}} \phi \equiv-\mathrm{i} \bar{S} \frac{\partial \phi}{\partial \boldsymbol{r}}=\boldsymbol{P}_{0} \phi
$$

From equation (15), the phase velocity of the plane wave is given as

$$
\boldsymbol{v}_{\text {phase }}=\frac{\omega_{0}}{\boldsymbol{k}_{0}}=\frac{H_{0}}{\boldsymbol{P}_{0}}
$$

Moreover, the phase factor in equation (13) can be rewritten using the magnitude of action, the Hamiltonian and the momentum of the free particle as,

$$
\phi(\boldsymbol{r}, t)=\mathrm{e}^{\mathrm{i} \theta}=\mathrm{e}^{\mathrm{i}\left(\boldsymbol{P}_{0} \boldsymbol{r}-H_{0} t\right) / \bar{S}} .
$$




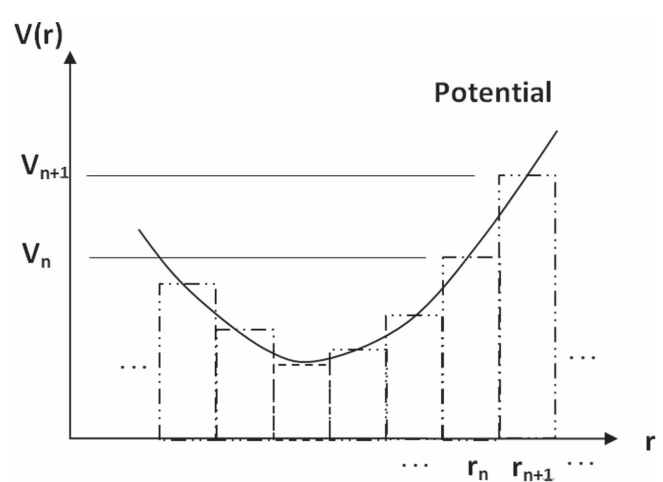

Figure 1. Approximation of a scalar potential field by small segments.

Using Euler's formula, the relation between the small variation of the phase angle $\Delta \theta$ and the small variation of the action $\Delta S$ can be deduced approximately,

$$
\Delta S=-\mathrm{i} \bar{S} \mathrm{e}^{\mathrm{i} \theta}\left(\mathrm{e}^{\mathrm{i} \Delta \theta}-1\right)=-\mathrm{i} \bar{S} \mathrm{e}^{\mathrm{i} \theta}(\cos \Delta \theta+\mathrm{i} \sin \Delta \theta-1)=\bar{S} \Delta \theta \mathrm{e}^{\mathrm{i} \theta},
$$

where the following approximations for small phase angle condition $(\Delta \theta \rightarrow 0)$ are used to derive the last equality in equation (19),

$$
\left(\frac{\sin \Delta \theta}{\Delta \theta}\right) \approx 1
$$

and

$$
\cos \Delta \theta \approx 1 \text {. }
$$

It should be noted that the action-phase relation presented in the literature such as [9-12], is $\Delta S=\hbar \Delta \theta$. However, it can be seen that equation (19) has an additional phase factor compared to the reference, as the action in equation (12) is a complex-value function. The magnitude of action variation is proportional to the phase angle. As pointed out in [11], there should be a constant with the unit of action if the action integral is considered as the phase angle. From equation (19), this constant seems to be the magnitude of action $\bar{S}$ that comes from the wave solutions of action in equation (12).

\subsection{Particle in a scalar potential field}

The governing equation of the particle in a scalar potential field can be deduced from the above plane wave solutions. For example, a massive particle that travels in a scalar potential field is considered. From the rectangular method in approximation theory, the scalar potential field can be approximated by small segments as shown in figure 1. At each small segment, the potential is approximated as constant and the free particle condition given in equations (1)-(3) is valid locally. Therefore, the corresponding Hamiltonian and momentum operator of the free particle at the small segment $n$ are,

$$
\mathrm{i} \bar{S} \frac{\partial \phi_{n}}{\partial t}=H_{n} \phi_{n}, \quad-\mathrm{i} \bar{S} \frac{\partial \phi_{n}}{\partial \boldsymbol{r}}=\boldsymbol{P}_{n} \phi_{n} .
$$

It should be noted that the expression of the wave-like solutions of the Hamilton and momentum of the particle should be consistent to the relations in classical particle dynamics (non-relativistic), 


$$
H_{n}=\frac{\boldsymbol{P}_{n}^{2}}{2 m}+V_{n}(\boldsymbol{r}) .
$$

Substituting the above relation into equation (22), one obtains the governing equation of the particle at segment $n$,

$$
\mathrm{i} \bar{S} \frac{\partial \phi_{n}}{\partial t}=\frac{-\bar{S}^{2}}{2 m} \frac{\partial^{2} \phi_{n}}{\partial \boldsymbol{r}^{2}}+V_{n}(\boldsymbol{r}) \phi_{n} .
$$

At the point between the adjacent small segments, free particle condition does not hold due to the potential force. However, if the length of segment is sufficiently small and the potential field is sufficiently smooth (e.g. quadratic function), the potential force becomes sufficiently small at the adjacent points. Therefore, the phase factors and their first and second spatial derivatives at the adjacent segments are approximately continuous, that is $\left|\phi^{\prime \prime}{ }_{n+1}-\phi^{\prime \prime}{ }_{n}\right|_{n \rightarrow \infty}<\epsilon$. If the potential function is piecewise smooth, the spatial derivative of the phase factor has discontinuities at the jump points. Nevertheless, within the smooth potential region, the index of the segment in the above equation can be dropped under the limitation of small segment, that is,

$$
\mathrm{i} \bar{S} \frac{\partial \phi}{\partial t}=\frac{-\bar{S}^{2}}{2 m} \frac{\partial^{2} \phi}{\partial \boldsymbol{r}^{2}}+V(\boldsymbol{r}) \phi .
$$

Moreover, the discretisation of the potential into small segments provides an intuitive picture of the potential force. From the property of complex exponential function, the new phase factor of the particle $\phi_{n+1}$ is equal to the multiplication of a phase factor $\Delta \phi$ (due to the step change of the potential) by the old phase factor of particle $\phi_{n}$,

$$
\phi_{n+1}=\phi_{n} \cdot \Delta \phi=\phi_{n} \cdot \mathrm{e}^{\mathrm{i}(\Delta k r-\Delta \omega t)},
$$

where

$$
\Delta \boldsymbol{k}=\boldsymbol{k}_{n+1}-\boldsymbol{k}_{n}, \quad \Delta \omega=\omega_{n+1}-\omega_{n}
$$

$\Delta \boldsymbol{k}$ and $\Delta \omega$ denote the change of the Hamiltonian and momentum due to the potential force, respectively. As the phase factor $\Delta \phi$ is linked to a free particle, the effect of potential force is linked to a potential particle. The process of interaction is similar to the local elastic collision of two free particles as in classical mechanics [3].

\subsection{Further extensions}

The results presented in sections 2.2 and 2.3 are based on the plane wave solutions of the dynamic variables. The linearity of the action wave equation enables the simple generalisation of the above results using the principle of superposition. From the principle of superposition, the general solution of the action in equation (11) can be expressed as the linear combination of the individual plane wave solutions,

$$
S=-\mathrm{i} \bar{S} \sum_{i=1}^{m} a_{i} \phi_{i}(\boldsymbol{r}, t)=-\mathrm{i} \bar{S} \varphi(\boldsymbol{r}, t),
$$

where

$$
\varphi(\boldsymbol{r}, t)=\sum_{i=1}^{m} a_{i} \phi_{i}(\boldsymbol{r}, t)
$$

is the general wave-like solution and $a_{i}$ is the normalised coefficients of the linear combination. Similarly, the general solutions of the Hamilton and momentum of the particle can be expressed as the linear combination of the plane wave solutions, 


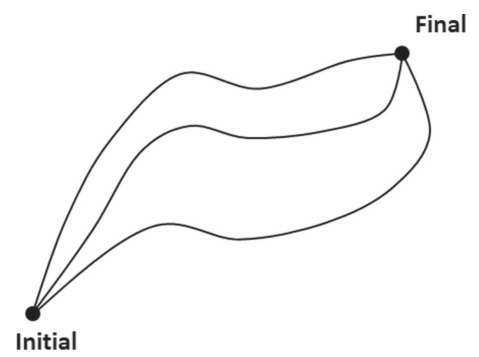

Figure 2. Propagation of action waves along different trajectories.

$$
H=\mathrm{i} \bar{S} \sum_{i=1}^{m} a_{i} \frac{\partial \phi_{i}}{\partial t}=\mathrm{i} \bar{S} \frac{\partial \varphi}{\partial t}, \quad \boldsymbol{P}=-\mathrm{i} \bar{S} \sum_{i=1}^{m} a_{i} \frac{\partial \phi_{i}}{\partial \boldsymbol{r}}=-\mathrm{i} \bar{S} \frac{\partial \varphi}{\partial \boldsymbol{r}} .
$$

Following similar procedures to those presented in section 2.3, the general form of the governing equation of a single particle in scalar potential field can be derived by substituting the above relations in equation (25),

$$
\mathrm{i} \bar{S} \frac{\partial \varphi}{\partial t}=\frac{-\bar{S}^{2}}{2 m} \frac{\partial^{2} \varphi}{\partial \boldsymbol{r}^{2}}+V(\boldsymbol{r}) \varphi, \quad \varphi=\varphi(\boldsymbol{r}, t) .
$$

Recalling Schrödinger's equation presented in the literature [9-12], the above equation is seen to have a similar form to time-dependent Schrödinger's equation in quantum mechanics. The phase factor $\varphi$ of the dynamic variable and the magnitude of action $\bar{S}$ serve similar roles as the wave function $\psi$ and the Planck's constant $\hbar$, respectively. It is also worth to note that the above equation is only valid in the smooth region of the potential field. At the point of discontinuity, the presented approach does not usually guarantee a general smooth solution. In order to obtain a general solution of the above equation, extra continuity conditions of the solution at each discontinuity point are necessary.

As can be seen from section 2, on one hand, dynamic variables such as action, Hamilton and momentum have wave-like solutions and therefore they have wave-like behaviour under time evolution. On the other hand, other variables such as time, displacement, velocity and acceleration of a particle do not have wave-like solutions. Therefore, those variables should not have wave-like behaviour. Since the particle is characterised via its dynamic variables, the wave picture of the particle is shown from only wave-like variables. Other variables do not contribute to the wave picture of the particle. It should be also noted that the wave-like behaviour of dynamic variables is intrinsic and does not rely on the presence of the external fields. This is the basis for the difference with classical fields such as acoustic waves. A detail discussion on this issue is presented in the following sections.

\section{Discussions}

\subsection{Maupertuis' principle and wave behaviour}

From classical mechanics, the trajectory of a conservative particle is generally determined from the principle of least action (Maupertuis' principle), which states that the particle chooses the trajectory that minimises the action between the initial and final positions, as shown in figure 2. For example, considering a free particle travelling from the initial to the final positions, since the solution of the action in equation (13) is time-invariant, the wavefront of the different paths have the same action when they arrive to the final position. If the 


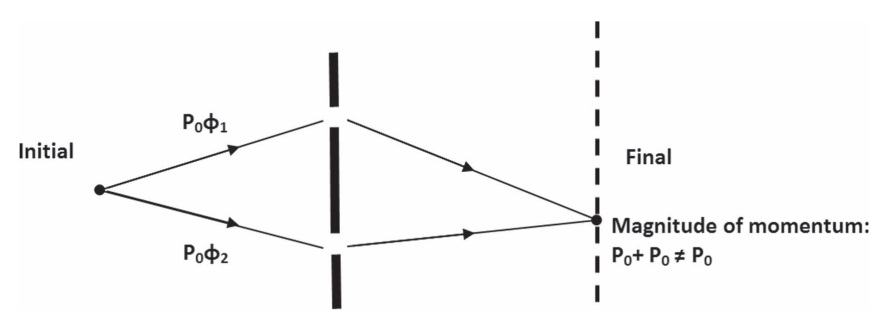

Figure 3. Superposition of wave-like variable based on a double-split experiment.

principle of least action is applied, the particle is allowed to take many distinct trajectories along the initial and final positions. It should be noted that parallel trajectories do not violate the Maupertuis' principle, since the principle does not require the trajectory to be unique but states that the allowable trajectory should have the minimum action value [1-3].

The wave-like solutions of the Hamiltonian and the momentum will propagate along different trajectories that are similar to the propagation of action waves. In detail, the wavefront of the Hamiltonian (or momentum) share the same value when they arrive to the final position. The only difference between the two waves along different trajectories is the time of travelling. However, if waves along different trajectories have enough time to reach the final position, the dynamic variables like Hamiltonian, momentum and action carried by these waves yield the same result. Since the particle is characterised via its dynamic variables, the wave-like variables that travel along different trajectories will contribute to the same picture of the particle at the final position.

The phenomenon of wave superposition is well-established in various fields of classical mechanics (e.g. acoustics [32]). However, it is worth to point out that the superposition of the above dynamic variables does not follow the rule of superposition of the classical waves (e.g. acoustic waves). Since the classical superposition involves both the magnitude and the phase of the variables, the magnitude of any superimposed variable (e.g. acoustic intensity) varies depending on the phase differences between the trajectories. On the other hand, the magnitudes of the dynamic variables of a free particle are required to be invariant through the time evolution. Figure 3 shows an example of superposition of wave-like variable based on a double-split experiment. According to the classical rule of superposition, the superimposed variable at bright finger has constructive interference and its magnitude is simply the sum of the two trajectories. If one applies the classical rule of superposition for wave-like variables such as momentum, it is easy to see that the magnitude of superimposed momentum will violate the conservation law since the sum of momentum from different trajectories (at final position) are not equal to momentum (at initial position).

\subsection{Comparisons to quantum mechanics}

Recalling from the literature such as [8], the quantum superposition rule states that the resultant wave function is the sum of the wave function from individual path. Applying the rule to the superposition of the wave-like dynamic variables, the magnitude of superimposed variable is preserved during the superposition. The phase factors from individual trajectory participate in the wave superposition. However, this does not change the magnitude of the superimposed variable at the final position. Besides the rule of superposition, technical similarities between the obtained results and the quantum relations are observed. For example, it can be seen that the expressions of the obtained wave-like solution and the wave function in quantum mechanics are similar. Table 1 compares the corresponding expressions of the 
Table 1. Comparison of the corresponding classical and quantum relations.

\begin{tabular}{ll}
\hline Classical relations: & Quantum relations: \\
\hline Phase factor (plane wave): & Wave function (plane wave): \\
$\phi(r, t)=\mathrm{e}^{\mathrm{i}(\boldsymbol{P r}-H t) / \bar{S}}$ & $\psi(r, t)=\mathrm{e}^{\mathrm{i}(\boldsymbol{P r}-H t) / \hbar}$ \\
Hamiltonian (plane wave): & Hamiltonian (plane wave): \\
$H=\bar{S} \omega$ & $H=\hbar \omega$ \\
Hamiltonian (operator): & Hamiltonian (operator): \\
$\hat{H}=\mathrm{i} \bar{S} \frac{\partial}{\partial t}$ & $\hat{H}=\mathrm{i} \hbar \frac{\partial}{\partial t}$ \\
Momentum (plane wave): & Momentum (plane wave): \\
$\boldsymbol{P}=\overline{\boldsymbol{S}} \boldsymbol{k}$ & $\boldsymbol{P}=\hbar \boldsymbol{k}$ \\
Momentum (operator): & Momentum (operator): \\
$\hat{\boldsymbol{P}}=-\mathrm{i} \bar{S} \frac{\partial}{\partial \boldsymbol{r}}$ & $\hat{\boldsymbol{P}}=-\mathrm{i} \hbar \frac{\partial}{\partial \boldsymbol{r}}$ \\
Governing equation: & Governing equation: \\
$\mathrm{i} \bar{S} \frac{\partial \varphi}{\partial t}=\frac{-\bar{S}^{2}}{2 m} \frac{\partial^{2} \varphi}{\partial \boldsymbol{r}^{2}}+V(\boldsymbol{r}) \varphi$ & $\mathrm{i} \hbar \frac{\partial \psi}{\partial t}=\frac{-\hbar^{2}}{2 m} \frac{\partial^{2} \psi}{\partial \boldsymbol{r}^{2}}+V(\boldsymbol{r}) \psi$ \\
\hline
\end{tabular}

constant, the dynamic variables and phase factor of a particle from the classical and the quantum relations.

From the table, the expressions in the classical relations have the same mathematical forms as the quantum relations. More specifically, the two sets of relations are connected from the following two substitutions,

$$
\bar{S} \leftrightarrow \hbar, \quad \varphi(\boldsymbol{r}, t) \leftrightarrow \psi(\boldsymbol{r}, t) .
$$

The differences between the two set of relations are also straightforward to observe. Firstly, it can be seen that the magnitude of action and Planck's constant serve similar roles in the corresponding relation. From section 2, the magnitude of action is an undetermined constant from the non-trivial plane wave solution. This quantity is well-defined in quantum mechanics as Planck's constant that measures the quanta. Moreover, it can be seen that the expression of the phase factor has the same structures as the particle wave function in Schrödinger's equation. The phase factor in classical relations plays a similar role to the particle wave function in quantum relations.

As the expressions in the left columns are directly based on classical mechanics, the above similarities show the connections between the classical and quantum relations. It is also worth to note that the above comparison mainly focuses on the formalism of wave mechanics presented in the standard text of quantum mechanics such as [10-12]. Just as there are parallel formalisms in classical mechanics, there are many parallel formalisms in quantum mechanics which are not discussed in this paper. The discussions of various parallel formalisms of quantum mechanics can be found in the literature such as [28, 29] and recently in [30].

\section{Conclusions}

This paper investigates the wave-like variables of a particle within the framework of classical mechanics in two steps. Firstly, from the free particle condition and Legendre transform, a linear homogenous wave equation of action is derived by using the two set of first-order relations simultaneously. The solutions of the dynamic variables of the particle are investigated from the wave equation. Dynamic variables such as action, Hamiltonian and 
momentum have been shown to be governed by wave-like solutions. The expressions of those variables have similar forms to expression in quantum mechanics. Other variables such as time, displacement, velocity and acceleration of a particle do not show the wave-like behaviour. With the variables solution obtained, it is also shown that the magnitude of action variation is proportional to the small variation of phase angle. Since a particle is assumed to be characterised via the dynamic variables, the wave-like dynamic variables provide an intrinsic wave-like picture of the particle.

The solutions obtained from free particle conditions are further extended to the situation of a conservative scalar potential for a single particle. Instead of using the substitution between action and phase angle in the Hamilton-Jacobi equation, a potential discretisation procedure is employed in the derivation. By dividing the potential field into small segments, the free particle equation is first setup. The obtained governing equation under smooth potential has a similar form to the Schrödinger equation. It should be noted that the obtained governing equation is only valid in the smooth potential region. The discretisation procedure also provides an intuitive link between the potential force and elastic collision in classical mechanics. Based on the principle of superposition, generalisations of solution of dynamic variables and corresponding governing equation of a particle are presented.

The rest of the paper discusses the obtained result under the framework of classical principle and its connections to quantum mechanics. The free particle case is considered under the Maupertuis' principle. For the wave solutions of action, it is found that there is more than one trajectory that can provide the minimum action. In fact, the propagation of wave-like variable under time evolution does not violate the classical principle. The contribution of waves from different trajectories leads to the superposition of the dynamic variables at the final position. On one hand, the superposition of the wave-like dynamic variables does not follow the rule of superposition of the classical waves. On the other hand, the quantum superposition rule provides a self-consistent interpretation of the superposition of the wave-like dynamic variables. The connections between the classical and quantum relations for corresponding variables are observed through direct comparison. Generally, the approach presented in this paper is mainly based on classical mechanics and it requires the audience at graduate and postgraduate level background.

\section{Acknowledgments}

The author is grateful to Dr S O Oyadiji for his helpful suggestions and proofreading of the manuscript. Additionally, the author also would like to acknowledge the useful comments from anonymous referees.

\section{References}

[1] Lanczos C 1970 The Variational Principles of Mechanics (New York: Dover)

[2] Gelfand I M and Silverman R A 2000 Calculus of Variations (New York: Dover)

[3] Taylor J R 2005 Classical Mechanics (Mill Valley, CA: University Science Books)

[4] Deriglazov A 2010 Classical Mechanics: Hamiltonian and Lagrangian Formalism (Berlin: Springer)

[5] Arnol'd V I 2013 Mathematical Methods of Classical Mechanics (Berlin: Springer)

[6] Heisenberg W 1958 Physics and Philosophy: The Revolution in Modern Science (London: Penguin Books Ltd)

[7] Messiah A 1965 Quantum Mechanics vol 1, 2 (Amsterdam: North-Holland)

[8] Feynman R P 1951 The concept of probability in quantum mechanics Proc. 2nd Berkeley Symp. on Mathematical Statistics and Probability pp 533-41 
[9] Omnes R 1994 The Interpretation of Quantum Mechanics (Princeton, NJ: Princeton University Press)

[10] Park D 2012 Introduction to the Quantum Theory (New York: Dover)

[11] Park D 2012 Classical Dynamics and its Quantum Analogues (Berlin: Springer)

[12] Shankar R 2012 Principles of Quantum Mechanics (New York: Springer)

[13] Bransden B H and Joachain C J 2000 Quantum Mechanics (Harlow: Pearson Education)

[14] Dirac P A M 1945 On the analogy between classical and quantum mechanics Rev. Mod. Phys. 17 195-9

[15] Feynman R P 1948 Space-time approach to non-relativistic quantum mechanics Rev. Mod. Phys. 20367

[16] Mukunda N 1974 Development of the analogy between classical and quantum mechanics Pramana 2 1-14

[17] Yourgrau W and Mandelstam S 1979 Variational Principles in Dynamics and Quantum Theory (New York: Dover)

[18] Leacock R A and Padgett M J 1983 Hamilton-Jacobi/action-angle quantum mechanics Phys. Rev. D 282491

[19] Prosser R T 1983 On the correspondence between classical and quantum mechanics: I. J. Math. Phys. 24 548-52

[20] Gutzwiller M C 1998 Resource letter ICQM-1: the interplay between classical and quantum mechanics Am. J. Phys. 66 304-24

[21] Dragoman D 2002 Phase space correspondence between classical optics and quantum mechanics Prog. Opt. 43 433-96

[22] Dragoman D and Dragoman M 2004 Quantum-Classical Analogies (Berlin: Springer)

[23] Carinena J F, Clemente-Gallardo J and Marmo G 2007 Introduction to quantum mechanics and the quantum-classical transition arXiv:0707.3539 [quant-ph]

[24] Masoliver J and Ros A 2009 From classical to quantum mechanics through optics Eur. J. Phys. 31171

[25] Field J H 2010 Derivation of the Schrödinger equation from the Hamilton-Jacobi equation in Feynman's path integral formulation of quantum mechanics Eur. J. Phys. 3263

[26] Nikolić H 2007 Quantum mechanics: myths and facts Found. Phys. 37 1563-611

[27] Landsman N P 2007 Between classical and quantum Handbook of the Philosophy of Science vol 2 (Amsterdam: North-Holland) pp 417-553

[28] Dirac P A 2013 Lectures on Quantum Mechanics (New York: Dover)

[29] Feynman R P, Hibbs A R and Styer D 2010 Quantum Mechanics and Path Integrals (New York: Dover)

[30] David F 2015 The Formalisms of Quantum Mechanics: An Introduction (Lecture Notes in Physics vol 893) (Berlin: Springer)

[31] Tikhonov A N and Samarskii A A 2013 Equations of Mathematical Physics (New York: Dover)

[32] Kinsler L E, Frey A R, Coppens A B and Sanders J V 1999 Fundamentals of Acoustics 4th edn (New York: Wiley) 